PHONON DETECTION BY THE FOUNTAIN PRESSURE

IN SUPERFLUID ${ }^{4}$ HELIUM FILMS

\title{
Wolfgang Eisenmenger
}

Physikalisches Institut, Universität Stuttgart

Pfaffenwaldring 57, D-7000 Stuttgart 80 , West Germany

\section{INTRODUCTION}

The importance of phonon focussing was first demonstrated by Taylor, Maris and Elbaum $|1|,|2|$, who calculated the phonon intensities for different modes in single crystals from the detailed form of the angle or $\mathrm{k}$-dependent sound velocity surfaces. The results of these calculations are well-confirmed by experiment. It is quite surprising that phonon focussing leads in several crystals to very narrow beams of phonon energy propagation especially for transverse modes, as recently calculated by Rösch and Weis $|3|$, who presented their computer results in a very instructive form, see Fig. 1. From these theoretical results it appears worthwhile to devise methods by which the intensity distribution of incoherent phonons propagating in single crystals can be measured in detail or directly imaged.

\section{EXPERIMENT}
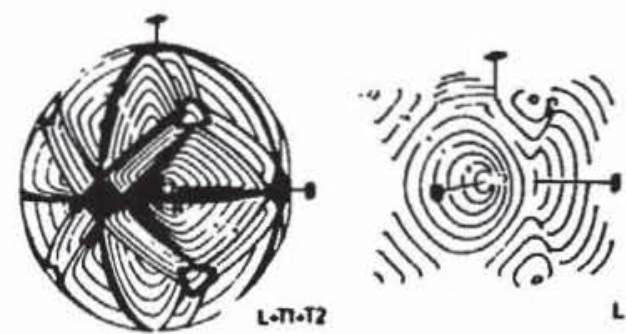

Whereas a distribution of phonon intensities in principle can be sampled by an array of bolometers or superconducting tunneling detectors, the possibility of an image converting system is also attractive for this purpose. In analogy to ultrasonic image converters based on the radiation pressure, it is the foun-
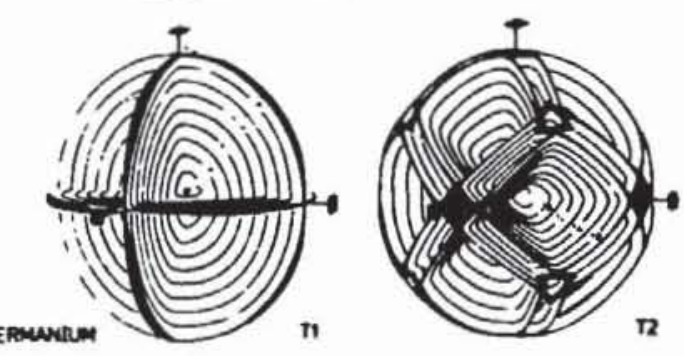

Fig. 1 Phonon Intensity Surfaces for Ge (After F. Rösch and 0 . Weis $|3|$ ) 
tain effect in liquid $4 \mathrm{He}$ below the $\lambda$-point that appears feasible for image converting of incoherent phonon distributions at low temperatures. The principle of this method is depicted in Fig. 2.

The single crystal, e.g. Si $|111|$; diameter $30 \mathrm{~mm}$, thickness $7 \mathrm{~mm}$ with a vacuum deposited constantan heater on its bottom side is immersed in liquid $4 \mathrm{He}$ below the $\lambda$-point, with the liquid $4 \mathrm{He}$ level a few $\mathrm{mm}$ below the upper crystal surface. Phonon emission from the heater increases the local temperature or phonon energy density in the superfluid film

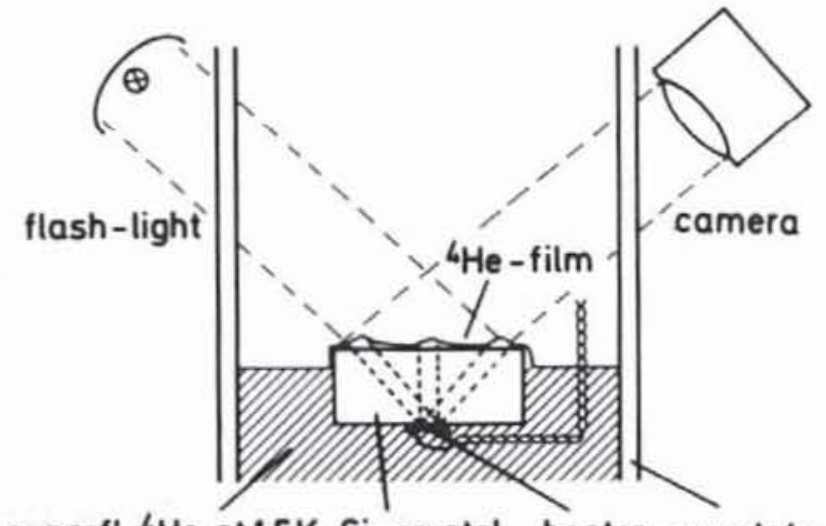

superfl. ${ }^{4} \mathrm{He}$ at $1.5 \mathrm{~K}, \mathrm{Si}$-crystal, heater, cryostate-wall

Fig. 2 Experimental Arrangement for Imaging Phonon Distributions by the Fountain Pressure covering the crystal surface in regions corresponding to directions of high phonon focussing. In these regions the fountain-pressure or the phonon radiation pressure, which numerically equals one third of the phonon energy density, leads to a locally increased film thickness. Under stationary conditions the film thickness is determined by the phonon energy density, whereas.the inflow of superfluid $4 \mathrm{He}$ from surface regions with lower temperature is balanced by the locally increased evaporation rate. The formation of a phonon intensity image by the fountain-pressure, therefore, depends critically on the condition that the evaporation rate from areas of increased film thickness does not exceed the inflow of superfluid $4 \mathrm{He}$ limited by the critical film flow velocity. Otherwise the film disappears by evaporation at the spots of highest intensities. If the critical film flow limit is increased by a somewhat rough crystal surface in addition to surface contaminations by condensed gases, higher phonon intensities, i.e. thicker films, are possible and the phonon surface structure becomes directly visible under oblique light incidence and can be photographed, as shown in Fig. 3. This phonon in-

Fig. 3 Phonon Distribution Image at the Surface of a $|111|$ Si crystal

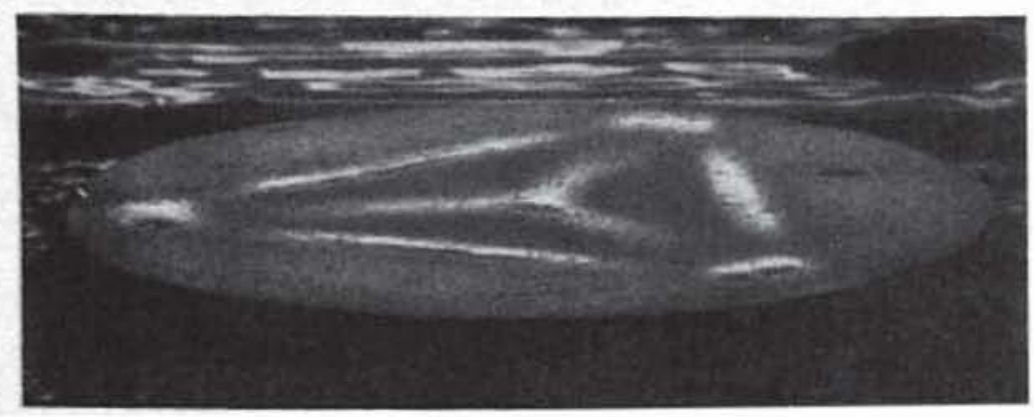


tensity picture has been obtained using a Si |111| crystal with the $4 \mathrm{He}$ level about $5 \mathrm{~mm}$ below the crystal surface. The heater power was 80 mWatts, and the bath temperature $1.5 \mathrm{~K}$; surface contamination was accomplished by condensed air, $1 \%$ air in the $4 \mathrm{He}$ gas of the cryostate before filling with liquid $4 \mathrm{He}$.

Fig. 3 has been obtained with the fountain pressure slightly exceeding the pressure corresponding to the liquid level difference between crystal surface and $4 \mathrm{He}$ bath. The heater power was applied for a time of approximately $1 \mathrm{sec}$, sufficient for the collection of enough $4 \mathrm{He}$ in the intensity maxima. The observed structure corresponds to the calculated distribution, c.f. Fig. 1 for Ge, clearly indicating the strong $|100|,|010|,|001|$ maxima, the $|111|$ maximum and the corresponding focussing planes of the slow transverse modes in addition to the focussing planes of the fast transverse modes. The splitting of the focussing planes of the slow transverse modes and the conical structure of the $|111| \max -$ imum in Fig. 1 are not resolved in the image Fig. 3. This possibly reflects the difference between phonon focussing in $\mathrm{Si}$ and $\mathrm{Ge}$.

With reduced liquid level difference pressure, the influence of the inclination of the crystal surface against the liquid $4 \mathrm{He}$ bath level becomes visible, as shown in Fig. 4.

Fig. 4 Phonon Distribution Image as Fig. 3 but with Reduced Liquid Level Difference and Inclined Surface.

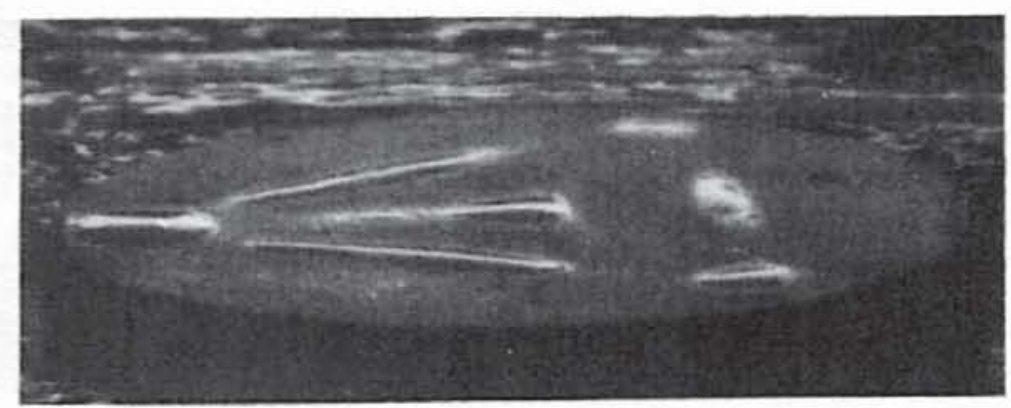

The $|100|$ maximum on the left side of the picture with a smaller liquid level difference has led to a strong drop-like collection of 4 He spreading over a larger area than the phonon beam width. This spreading corresponds to the finite lateral heat conduction in a thick $4 \mathrm{He}-\mathrm{layer}$, and reduces the phonon energy density in the film.

In principle, it is also possible to use fountain pressures below the liquid level difference. Under this condition, film thickness changes are of the order of $1000 \AA$ and it is necessary to use schlieren-, interference- or ellipsometric techniques to visualize phonon-surface structures. 


\section{QUANTITATIVE DISCUSSION}

Film thickness under the influence of the fountain pressure

The binding potential $\Phi$ of $4 \mathrm{He}$ atoms in the film surface can be described by c.f. $|4|$

$$
\Phi=-a / d^{3}
$$

$\alpha=$ van der Waals constant for 4 He atoms of the film bound to the substrate, $d=$ film thickness.

Under equilibrium conditions $\Phi$ equals $-m g(h+d),(m=$ mass of He atoms, $\mathrm{g}=$ gravitational acceleration, $\mathrm{h}=$ liquid level difference with respect to crystal surface), the potential energy of $\mathrm{He}$ atoms at the liquid He level $\mathrm{h}$ below the crystal surface. Alternatively, the equilibrium condition can be described by an effective van der Waals pressure, which tends to increase the film thickness and is counterbalanced by the liquid level difference pressure $P_{L}=\rho_{I} g(h+d) ;\left(\rho_{L}=\right.$ density of liquid $\left.4 \mathrm{He}\right)$. This leads to the film thickness expression:

$$
P_{L}=c / d^{3} \text { with } c=\rho_{L} \alpha / m
$$

Taking account of the fountain pressure and spatial variations of the film thickness, $P_{L}$ has to be replaced by the total pressure $\mathrm{P}_{t}$ leading to the relation:

$$
P_{t}=P_{L}-P_{F}+P_{S}=c / d^{3}
$$

with $\mathrm{P}_{\mathrm{F}}=$ fountain pressure and $\mathrm{P}_{\mathrm{S}}=$ pressure by surface tension under the film surface curvature. In evaluating Equ. (3) with respect to the film thickness two limiting situations have to be considered:

i.) $\left|P_{F}\right|<\left|P_{L}\right|$ : In this regime of "low" fountain pressure the film thicknesS increase $\Delta d$ (neglecting $\mathrm{P}_{\mathrm{S}}$ ) results in the approximative relation:

$$
\Delta d=d_{0} P_{F} / 3 P_{L}
$$

$\mathrm{d}_{0}=$ film thickness without fountain pressure.

ii.) $\left|P_{F}\right|>\left|P_{L}\right|$ : In this region of "high" fountain pressure the film thickness $\mathrm{d}$ is approximated by

$$
\mathrm{d}=\left\{\left(\mathrm{P}_{\mathrm{F}}-\mathrm{P}_{\mathrm{S}}\right) / \mathrm{P}_{\mathrm{L}}-1\right\} \cdot \mathrm{h}
$$

Estimates of $\mathrm{P}_{\mathrm{S}}$ for the experimentally observed film structures indicate that surface tension influences can be neglected.

Film temperature and fountain pressure

The experimental temperature dependence of the fountain pressure $14 \mid$ can be described by:

$$
\mathrm{P}_{\mathrm{F}}=593 \mathrm{~T}^{3} \cdot \Delta \mathrm{T}
$$

$\mathrm{PF}$ in $\mathrm{cm}$ liquid $4 \mathrm{He}, \mathrm{T}=$ bath temperature and $\Delta \mathrm{T}=$ film excess 
temperature in $\mathrm{K}$. The prefactor corresponds to a measurement at $1.5 \mathrm{~K}$ and exceeds the pure phonon contribution by a factor of 2 as consequence of roton excitations and the curvature of the phonon dispersion curve. Equ. (6) indicates that a temperature increase in the film of $\Delta \mathrm{T}=10^{-4} \mathrm{~K}$ at a bath temperature of $1.5 \mathrm{~K}$ results in a fountain pressure $\mathrm{P}_{\mathrm{F}}=2 \mathrm{~cm}$ liquid $4 \mathrm{He}$. In calculating $\Delta \mathrm{T}$ under phonon irradiation we use the balance between phonons transmitted from the crystal into the film against the energy transport by excess $4 \mathrm{He}$ atoms evaporating from the film and phonons backradiated from the film into the crystal. The detailed analysis shows that direct heat conduction from the film into the $4 \mathrm{He}$ gas can be neglected in comparison to the energy transport according to the latent heat of evaporation. Also lateral heat conduction within the liquid $4 \mathrm{He}$ film has little influence unless the film thickness approaches the width of the structure. Using the rates of He atoms incident from the vapor (sticking probability 1) which are in equilibrium with $4 \mathrm{He}$ atoms evaporated from the liquid film, the excess evaporation rate at the elevated temperature $T+\Delta T$ of the liquid film and the corresponding energy transport by the latent heat of evaporation can be calculated. This, together with the phonon backradiation (assuming $20 \%$ transmission within the angle limit of total reflection), leads to the total energy transport $\dot{q}$ as function of $\Delta T$ :

$$
\dot{q}=\left\{5.94 \cdot 10^{4} \cdot \mathrm{T}^{-2} \cdot 5 \cdot \exp -Q / R T+1.39 \mathrm{~T}^{3}\right\} \cdot \Delta \mathrm{T}
$$

$\dot{\mathrm{q}}$ measured in Watt $/ \mathrm{cm}^{2}, \mathrm{~T}$ and $\Delta \mathrm{T}$ in $\mathrm{K} . \mathrm{R}=8.3 \mathrm{Joule} / \mathrm{Mole} \cdot \mathrm{K}$, ideal gas constant. $Q=82 \mathrm{Joule} / \mathrm{Mole}$, latent heat of evaporation in the $1 \mathrm{~K}$ range.

In the calculation the latent heat of evaporation from the film has been set equal to the latent heat of evaporation from the free liquid surface. In thermodynamic equilibrium only the difference in potential energy $\mathrm{mg}(\mathrm{h}+\mathrm{d})$ has to be taken into account, which is small compared to the heat of evaporation under the conditions of the experiment. Furthermore, the energy contribution by transport entropy, i.e. the energy necessary to heat the inflowing superfluid component to the film temperature $T+\Delta T$ can be neglected.

Combining Equations (6) and (7) leads to the relation between the fountain pressure and the phonon intensity transmitted into the film. At the bath temperature of $1.5 \mathrm{~K}$ and the transmitted phonon intensity of $1 \mathrm{mWatt} / \mathrm{cm}^{2}$ this results in

$$
\mathrm{P}_{\mathrm{F}}=5.8 \times 10^{-2} \mathrm{~cm} \text { liquid } 4 \mathrm{He}
$$

corresponding to $20 \%$ phonon backradiation and $80 \%$ evaporation. Therefore, with a liquid level $0.5 \mathrm{~cm}$ below the crystal surface the condition $\mathrm{P}_{\mathrm{F}}>\mathrm{P}_{\mathrm{L}}$ for a direct observation of the phonon intensity structures requires a transmitted phonon intensity of $I_{\text {pht }}=8.6 \mathrm{mWatt} / \mathrm{cm}^{2}$. For a phonon transmission factor of $20 \%$ at the crystal - film boundary this corresponds to an incident phonon 
intensity of $43 \mathrm{mWatt} / \mathrm{cm}^{2}$. In the experiment (c.f. Fig. 3) a total heater power of $80 \mathrm{mWatt}$ has been applied. A rough estimate based on the crystal dimensions, using a phonon focussing factor of 10 and assuming that $2 / 3$ of the heater power is directly emitted into the liquid He bath, results in the incident phonon intensity of $50 \mathrm{mWatt} / \mathrm{cm}^{2}$. The comparison with the calculated minimum intensity of $43 \mathrm{mWatt} / \mathrm{cm}^{2}$ indicates satisfactory agreement between experiment and theoretical model.

Intensity limit by the critical film flow

With increased transmitted phonon intensity and evaporation rate the liquid He mass transport becomes higher than allowed by the critical film flow. Considering a narrow maximum of the phonon intensity distribution in the film of $1 \mathrm{~mm}$ by $1 \mathrm{~mm}$, the critical film flow of $8 \times 10^{-5} \mathrm{~cm}^{2} \mathrm{sec}^{-1}|4|$ for clean polished surfaces corresponds to an evaporation heat flow of $\dot{q}=9.5 \mathrm{mWatt} / \mathrm{cm}^{2}$, which is close to the transmitted phonon intensity $I_{\mathrm{pht}}=8.6 \mathrm{~mW} / \mathrm{cm}^{2}$ considered before as necessary for obtaining a directly visible image of the phonon structure. For contaminated surfaces the critical film flow can be increased by one order of magnitude. In our experiments (c.f. Fig. 3) the surface contamination turned out to be very important. In experiments with a $|100|$ oriented Si crystal the disappearance of the He film in the $|100|$ phonon intensity maximum demonstrated that the critical film flow condition is easily reached with extreme differences in phonon focussing.

I gratefully acknowledge the technical assistance of Mrs. S. Döttinger, whose excellent experimental and photographic skill has led to the presented phonon distribution images.

REFERENCES :

1. B. Taylor, H. J. Maris and C. Elbaum, Phys. Rev. Lett. 23, 416, (1969).

2. B. Taylor, H. J. Maris and C. Elbaum, Phys. Rev. B 3, 1462 , (1971)

3. F. Rösch and 0. Weis, Z. Phys. B 25, 115, (1976)

4. J. Wilks, "The Properties of Liquid and Solid Helium", Clarendon Press, Oxford (1967) 